\title{
Association of ultrarapid freezing of mouse oocytes with increased polyploidy at the pronucleate stage, reduced cell numbers in blastocysts and impaired fetal development
}

\author{
J. Van der Elst, S. Nerinckx and A. C. Van Steirteghem \\ Centre for Reproductive Medicine, University Hospital, Dutch-speaking Brussels Free University, \\ Laarbeeklaan 101, 1090 Brussels, Belgium
}

\begin{abstract}
Mature mouse oocytes were frozen ultrarapidly with a cryoprotectant solution consisting of $3.5 \mathrm{~mol}$ dimethylsulfoxide $1^{-1}$ and $0.5 \mathrm{~mol}$ sucrose $1^{-1}$ or exposed to the freezing solution and recovered. Freshly collected oocytes were used as controls. After ultrarapid freezing and thawing, a high mean percentage of oocytes (78\%) survived. The incidence of parthenogenetic activation in frozen-thawed oocytes was not increased. After insemination in vitro, the rate of two-cell formation was decreased (59\% versus $69 \%$ ). Examination of the chromosome complement of first-cleavage embryos revealed that the incidence of polyploid embryos was four times that of the control group ( $23 \%$ versus $6 \%$ ). Fewer fertilized eggs progressed to the blastocyst stage ( $49 \%$ versus $81 \%$ ) and the mean number of cells per blastocyst was decreased. Furthermore, the capacity of transferred blastocysts to develop in vivo was reduced. Autopsy at day 17 of gestation revealed that the proportion of embryos implanting was lower in the embryos derived from ultrarapidly frozen-thawed oocytes. Furthermore, some of the living fetuses were abnormal, but our sample size is too small for the effect to be significant. In the solution control group, no differences were found compared with the control group. Although the study needs further results to draw definite conclusions, our findings question whether the applied protocol is suitable for mouse oocytes.
\end{abstract}

\section{Introduction}

The determination of reliable methods for the cryopreservation of oocytes is still a challenging area. The interest in oocyte freezing is particularly related to the use of ovarian hyperstimulation regimens in assisted reproductive medicine and the subsequent recovery of an excess of oocytes. The availability of oocyte freezing would solve problems of desynchronization in donation schedules and alleviate the ethical objections and legal problems involved in frozen embryo storage and disposal. Furthermore, the original goal of saving oocytes from patients facing imminent loss of ovarian function would also be served.

The possibility of freezing mouse oocytes to $-196^{\circ} \mathrm{C}$ and thawing them again to permit the birth of live young was first described by Whittingham (1977). Since then, numerous attempts at oocyte freezing using slow cooling procedures have been consistently unsuccessful in terms of both efficiency and safety. There are problems of overall reduced survival and fertilization rates (Glenister et al., 1987; Trounson and Kirby, 1989), zona hardening (Johnson et al., 1988; Johnson, 1989; Carroll et al., 1990; Vincent et al., 1990a, b, 1991; Wood et al., 1992), incomplete reversibility of spindle disassembly (Magistrini and Szöllösi, 1980; Johnson and Pickering, 1987; Pickering and Johnson, 1987; Van der Elst et al., 1988; Pickering et al., 1990; Aigner et al., 1992) and higher incidence of polyploidy (Wood,

Received I July 1992.
1986; Al-Hasani et al., 1987; Glenister et al., 1987), thus the cryopreservation of mature oocytes by slow conventional cooling techniques cannot be recommended. The human oocyte in particular seems unsuited to cryopreservation. Only seven pregnancies followed by five live births have been reported over 7 years (Chen, 1986, 1989; Al-Hasani et al., 1987; van Uem et al., 1987; Kolodziej et al., 1991). Most such clinical programmes have been stopped.

Surrey and Quinn (1990) reported the successful cryopreservation of mouse oocytes by ultrarapid freezing. These authors report a high mean percentage of normal oocytes after thawing $(61 \%)$ as well as high rates of two-cell formation $(77 \%)$ and oocyte-to-blastocyst development $(59 \%)$. The value of the ultrarapid freezing approach has been demonstrated for mouse preimplantation embryos, as under optimal conditions it allows cyropreservation without a significant loss of viability in vitro or in vivo, or any demonstrable chromosomal defects (Trounson et al., 1987, 1988; Shaw et al., 1991a, b). Ultrarapid freezing of human embryos has so far resulted in human pregnancies and deliveries (Gordts et al., 1990; Feichtinger et al., 1991).

In a further attempt to evaluate the ultrarapid freezing technique for mouse oocytes, we found it worthwhile to repeat and to extend the studies performed by Surrey and Quinn (1990). We investigated not only in vitro developmental competence but also the incidence of parthenogenetic activation, the chromosome complement of first-cleavage embryos, the cell number and mitotic index in day 5 blastocysts and the in vivo 
developmental potential of transferred blastocysts. In a previous study we reported that ultrarapid freezing preserves spindle morphology better than does slow freezing (Aigner et al., 1992).

\section{Materials and Methods}

\section{Oocyte collection}

Unfertilized oocytes were collected from 4- to 6-week-old (C57Bl/6) $\times$ CBAca) $\mathrm{F}_{1}$ hybrid mice that had undergone ovarian hyperstimulation by i.p. injections of 5 iu of pregnant mares' serum gonadotrophin (PMSG; Folligon: Intervet, The Netherlands) and 5 iu of hCG (Chorulon: Intervet, The Netherlands) administered $48 \mathrm{~h}$ apart. The animals were killed 14-15 h after administration of hCG. Postovulatory oocytes were released in M2 (Quinn et al., 1982) containing $5 \mathrm{mg} \mathrm{BSA}$ $\mathrm{ml}^{-1}$ (Sigma Co., St Louis, MO). Cumulus dispersion was obtained in this medium with $1 \mathrm{mg}$ hyaluronidase $\mathrm{ml}^{-1}$ (type IS; Sigma $C_{0}$.). Thereafter, oocytes were washed twice in M2 with $5 \mathrm{mg} \mathrm{BSA} \mathrm{ml}^{-1}$. Between 5 and $10 \%$ of oocytes lacking a clearly visible polar body were discarded after inspection under a Wild stereomicroscope at a magnification of $\times 50$. During the period in which the experiments were performed, a day-to-day variation in the quality of the recovered oocytes was recorded. When more than $25 \%$ of the oocytes revealed abnormalities, in vitro fertilization and freezing were not attempted. The most frequently observed abnormalities were dark cytoplasmic granulation, enlargement of the perivitelline space and fragmentation.

\section{Experimental design}

Experiments were performed in four different sets. In the first set, the developmental potential of ultrarapidly frozen oocytes in vitro was investigated to repeat the study of Surrey and Quinn (1990). Survival rate as well as two-cell and blastocyst formation rates were recorded. In addition, we prepared spreads of the day 5 blastocysts obtained to count the number of cells and mitoses. In the three other independent sets of experiments: (1) the incidence of parthenogenetic activation, (2) the chromosome complement of first-cleavage embryos and (3) the developmental potential of blastocysts in vivo after transfer were investigated. In the four sets of experiments, solution controls exposed to the freezing mixture were included where indicated. However, as ultrarapid freezing and solution control experiments were not performed on the same day, they were each compared with their respective control oocytes. Fresh oocytes that were collected near the middle of the $1 \mathrm{~h}$ recuperation time of the thawed oocytes were used as controls for frozen-thawed oocytes. As controls for exposed oocytes, their counterparts that had not been exposed to the freezing solution but were kept at $37^{\circ} \mathrm{C}$ between the times of collection and insemination were used so that the results of the different sets of experiments could be related; only experiments for which the survival rate and in vitro developmental potential were not different from the initial findings in the first set of experiments on in vitro development were included.

\section{Ultrarapid freezing and thawing}

Oocytes were frozen according to a protocol described by Surrey and Quinn (1990). Manipulations were carried out in $35 \mathrm{~mm}$ plastic Petri dishes at an ambient temperature of $22^{\circ} \mathrm{C}$. All freezing solutions were made with $\mathrm{M} 2$ containing $20 \%$ bovine calf serum (BCS; Hyclone Laboratories, Logan, Utah). Groups of 25-30 oocytes were frozen together. Briefly, oocytes were partially dehydrated in $0.25 \mathrm{~mol}$ and $0.50 \mathrm{~mol}$ sucrose $1^{-1}$ for $5 \mathrm{~min}$ each. The oocytes were then exposed to a mixture of $3.50 \mathrm{~mol}$ dimethylsulfoxide $\mathrm{l}^{-1}$ (DMSO) and $0.50 \mathrm{~mol}$ sucrose $\mathrm{I}^{-1}$ for a total of $2.5 \mathrm{~min}$. Plastic $500 \mu \mathrm{l}$ straws (Instruments de Médicine Vétérinaire (I.M.V.), l'Aigle) were loaded as follows: $100 \mu \mathrm{l}$ freezing medium, a $100 \mu \mathrm{l}$ air bubble, $100 \mu \mathrm{l}$ freezing medium containing the oocytes, a $100 \mu \mathrm{l}$ air bubble and $100 \mu \mathrm{l}$ of freezing medium. The straw was plugged with polyvinyl alcohol powder (I.M.V., l'Aigle, France) and left horizontally on the bench of a laminar flow hood. At the end of the $2.5 \mathrm{~min}$ equilibration time the straws were plunged directly into liquid nitrogen. Thawing was achieved by agitating straws in a $37^{\circ} \mathrm{C}$ water bath for 5-6 s until the solution melted. The content of the straws was emptied into a $35 \mathrm{~mm}$ Petri dish containing $2 \mathrm{ml} \mathrm{M} 2$ with $20 \% \mathrm{BCS}$ and $0.50 \mathrm{~mol}$ sucrose $\mathrm{l}^{-1}$. After $5 \mathrm{~min}$, the oocytes were transferred to medium containing $0.25 \mathrm{~mol}$ sucrose $1^{-1}$. Removal of the sucrose was achieved by washing twice for $5 \mathrm{~min}$ in $\mathrm{M} 2$ with $5 \mathrm{mg} \mathrm{BSA} \mathrm{ml}^{-1}$. Solution controls underwent an identical treatment except that they were not loaded into straws and frozen-thawed.

\section{Assessment of morphological survival rate}

Oocyte morphology was assessed under a Wild stereomicroscope at a magnification of $\times 50$ immediately after dilution of the cryoprotectants. Oocytes were considered damaged when the cytoplasm was dark and granular or when the perivitelline space was enlarged. Oocytes of normal appearance were transferred to culture medium M16 (Quinn et al, 1982) with $5 \mathrm{mg}$ crystalline BSA ml ${ }^{-1}$ (ICN, Bucks) under oil in a humidified $5 \%$ $\mathrm{CO}_{2}$ atmosphere at $37^{\circ} \mathrm{C}$. The oocytes were assessed again $\mathrm{Ih}$ after thawing or exposure. The numbers of damaged oocytes of both assessments were added and the percentage of normal oocytes referred to in the tables results from this aggregate figure.

\section{Assessment of in vitro two-cell and blastocyst formation rates}

After $1 \mathrm{~h}$ of recuperation following thawing or exposure, oocytes were inseminated with approximately $1.5 \times$ $10^{6}$ spermatozoa ml $\mathrm{m}^{-1}$ in Whittingham's medium with $30 \mathrm{mg}$ $\mathrm{BSA} \mathrm{ml} \mathrm{m}^{-1}$. The oocyte age at the moment of insemination was approximately $15.30 \mathrm{~h}$ after hCG treatment: collection at $14 \mathrm{~h}$ after hCG plus $30 \mathrm{~min}$ freezing-thawing or exposure procedure plus $1 \mathrm{~h}$ of recuperation. Spermatozoa were obtained from the distal caudae epididymides of male $F_{1}$ hybrid mice of proven fertility. After $4 \mathrm{~h}$ of insemination, oocytes were washed five times in M16 with $5 \mathrm{mg} \mathrm{BSA} \mathrm{ml}^{-1}$. Embryo culture was continued in M16 with $5 \mathrm{mg}$ crystalline BSA ml${ }^{-1}$. The fertilization rate was assessed as the number of two-cell embryos at $24 \mathrm{~h}$ after insemination compared with the number of oocytes inseminated. 
Development was scored as the number of blastocysts at $120 \mathrm{~h}$ after insemination compared with the number of two-cell embryos previously recorded.

\section{Number of nuclei and mitotic index in day 5 blastocysts}

Blastocysts were fixed according to the method of Tarkowski (1966). Blastocysts were exposed to a hypotonic $2 \%$ citrate solution for $6 \mathrm{~min}$ at $22^{\circ} \mathrm{C}$ and transferred in a small droplet of citrate to the surface of a clean glass slide. Fixation was done with a mixture of methanol:acetic acid (3:1), droplets of which were placed on top of the blastocysts using a finely drawn pipette. The attachment of embryos to the slide was followed under a stereomicroscope. Slides were stained with a $10 \%$ Giemsa solution for $10 \mathrm{~min}$ at $22^{\circ} \mathrm{C}$, rinsed in MilliQ water and air-dried. Microscopy was performed with a Leitz Dialux 22 microscope at a magnification of $\times 400$. Blastocysts were scored blind by two independent investigators. The number of Giemsa-stained nuclei was counted on blastocysts with their nuclei clearly in juxtaposition. Blastocysts were grouped in four categories chosen according to the expected number of blastomeres after five, six and seven cycles of cell division: less than 32 , from 32 to 64 , from 64 to 128 and more than 128 . The number of mitoses per blastocyst was also recorded on the original spreads and was transformed into a mitotic index per blastocyst, being the number of mitotic nuclei divided by the total number of nuclei per blastocyst.

\section{Parthenogenetic activation of oocytes}

In a first separate study, we investigated parthenogenetic activation of oocytes after ultrarapid freezing or exposure to the ultrarapid freezing solution. Surviving oocytes were screened for parthenogenetic activation by examining for the presence of polar bodies and pronuclei under Nomarski optics $5-6 \mathrm{~h}$ after thawing. Four categories of oocytes can be distinguished according to Kaufman (1983): (1) nonactivated oocytes: metaphase Il oocytes with the first polar body extruded (visible or degenerated) and no pronuclei present; (2) activated metaphase II oocytes with extrusion of the second polar body and one pronucleus; (3) activated metaphase II oocytes with retention of the second polar body and containing one or two pronuclei; and (4) activated metaphase II oocytes with two equally sized blastomeres, each with one nucleus. This is called immediate cleavage.

\section{Chromosome analysis of first-cleavage embryos}

In a second separate study, we examined the chromosome complement of in vitro fertilized oocytes before their first cleavage division. Eggs were recovered from the insemination droplet and transferred to M16 medium with $5 \mathrm{mg}$ crystalline BSA ml${ }^{-1}$ and with $0.2 \mu \mathrm{g}$ colcemide $\mathrm{ml}^{-1}$. After overnight culture, these zygotes were fixed according to the method of Tarkowski (1966) as described above. Microscopy was performed with a Leitz Dialux 22 at a magnification of $\times 1000$. Metaphases were scored blind by two independent investigators. For the embryos with two or more chromosome sets, the ploidy was added giving a distribution with diploid, aneuploid and polyploid embryos.

\section{Transfer of blastocysts to pseudopregnant foster mice}

In a third separate study, the in vivo developmental capacity of embryos cultured in vitro to the early blastocyst stage was assessed by uterine transfer. Recipient females were made pseudopregnant by mating with sterile vasectomized Swiss males. The presence of a copulation plug on the next morning was considered as the mark for the first day of pseudopregnancy. The transfer was performed on the third day of pseudopregnancy with day 4 in vitro developed blastocysts. Animals were anaesthetized by i.p. injection of $0.1 \mathrm{ml}$ of a $3.3 \%$ Nembutal solution. The uterine horns were expelled through a small midline incision. Five blastocysts were guided into each uterine horn with the aid of a finely drawn pipette. Control blastocysts were transferred to the right horn, while blastocysts from the experimental group were transferred to the left horn. On day 17 of gestation, autopsies were performed. Fetuses were examined for the presence of gross morphological abnormalities.

\section{Statistical analysis}

Data for each separate study were obtained from four to nine replicate experiments. Frequencies obtained for categorical parameters were compared using a $\chi^{2}$ test. Mean values were compared with Student's $t$ test. Both tests were used as provided in the Apple Macintosh Statview 512+ package.

\section{Results}

\section{Morphological survival rate}

In total 362 oocytes were recovered from freezing (Table 1 ), that is $89 \%$ of the original number of frozen oocytes. A mean survival rate of $78 \%$ was recorded with a variation from $45 \%$ to $92 \%$ between individual straws. Most of the abnormal oocytes were of the degenerative type and were found in the first assessment immediately after thawing. After exposure to the freezing solution, almost all oocytes were morphologically normal.

\section{Two-cell and blastocyst formation rates}

Insemination of 340 freshly collected oocytes resulted in the formation of a mean of $69 \%$ of two-cell embryos $24 \mathrm{~h}$ after insemination (Table 1). Of these, $81 \%$ developed to the blastocyst stage at $120 \mathrm{~h}$ after insemination. Fewer ultrarapidly frozen oocytes developed in vitro to the same stages: $59 \%$ two cells $\left(P=0.028 ; \chi^{2}=4.83\right)$ and $49 \%$ blastocysts $\left(P=0.001 ; \chi^{2}=\right.$ 32.09). After exposure of oocytes to the ultrarapid freezing solution, there was no decrease of the two-cell formation rate ( $74 \%$ versus $77 \%$ in their controls) but the percentage of two cells developing to blastocysts was lower compared with that of controls ( $53 \%$ versus $\left.68 \% ; P=0.008 ; \chi^{2}=6.95\right)$.

\section{Number of nuclei per blastocyst}

On inspection by stereomicroscope, blastocysts from the experimental group exhibited a normal spherical shape and 
Table 1. Survival rate and in vitro two-cell and blastocyst formation rates of mouse oocytes following ultrarapid freezing or exposure to a $3.5 \mathrm{~mol}$ dimethylsulfoxide $\mathrm{I}^{-1} / 0.5 \mathrm{~mol}$ sucrose $\mathrm{l}^{-1}$ ultrarapid freezing solution

\begin{tabular}{|c|c|c|c|c|c|}
\hline Treatment & $\begin{array}{l}\text { Number of } \\
\text { recovered } \\
\text { oocytes }\end{array}$ & $\begin{array}{l}\text { Number of } \\
\text { normal oocytes } \\
\text { (\% of oocytes } \\
\text { recovered) }\end{array}$ & $\begin{array}{c}\text { Number of } \\
\text { inseminated } \\
\text { oocytes }\end{array}$ & $\begin{array}{c}\text { Number of } \\
\text { two-cell embryos } \\
\text { (\% of inseminated } \\
\text { oocytes) }\end{array}$ & $\begin{array}{c}\text { Number of } \\
\text { blastocysts } \\
\text { (\% of two cells) }\end{array}$ \\
\hline Control & 347 & $340(98 \%)$ & 340 & $233(69 \%)$ & $157 / 193(81 \%)$ \\
\hline Ultrarapid freezing ${ }^{*}$ & 362 & $281(78 \%)$ & 241 & $143(59 \%)^{\mathrm{d}}$ & $51 / 104(49 \%)^{b}$ \\
\hline Control & 185 & $181(98 \%)$ & 181 & $139(77 \%)$ & $95(68 \%)$ \\
\hline Exposure $^{*}$ & 187 & $176(94 \%)$ & 176 & $13 \mathrm{I}(74 \%)$ & $69(53 \%)^{c}$ \\
\hline
\end{tabular}

ap $=0.028$ compared with controls, $\chi^{2}=4.83$, d.f. $=1$.

${ }^{b} P=0.001$ compared with controls, $\chi^{2}=32.09$, d.f. $=1$.

$c P=0.008$ compared with controls, $\chi^{2}=6.95$, d.f. $=1$.

*Both sets of experiments were not performed contemporarily.

Ultrarapid freezing: nine replicate experiments, one to three replicate straws per experiment; exposure: five replicate experiments.

Table 2. Classification of blastocysts according to the number of nuclei $(x)$. Categories are based on the expected number of nuclei after the 5 th, 6 th and 7 th cleavage. Blastocysts were derived from oocytes that were ultrarapidly frozen or were exposed to a $3.5 \mathrm{~mol}$ dimethylsulfoxide $1^{-1} / 0.5 \mathrm{~mol}$ sucrose $\mathrm{l}^{-1}$ ultrarapid freezing solution. The mitotic index per category is indicated in italic

\begin{tabular}{|c|c|c|c|c|c|c|}
\hline \multirow[b]{2}{*}{ Treatment } & \multirow[b]{2}{*}{ Blastocysts } & \multirow{2}{*}{$\begin{array}{c}\text { Mean } \pm \text { SD } \\
\text { number of nuclei }\end{array}$} & \multicolumn{4}{|c|}{$\begin{array}{c}\text { Number }(\%) \text { of blastocysts with } \\
\text { MI }\end{array}$} \\
\hline & & & $x \leqslant 32$ & $32<x \leqslant 64$ & $64<x \leqslant 128$ & $x>128$ \\
\hline \multirow[t]{2}{*}{ Control } & 126 & $70 \pm 34$ & $16(13)$ & $51(40)$ & $53(42)$ & $6(5)$ \\
\hline & & & $5.5 \%$ & $3.2 \%^{e}$ & $2.3 \%$ & $1.7 \%$ \\
\hline \multirow[t]{2}{*}{ Ultrarapid freezing $^{* a}$} & 52 & $38 \pm 23^{b, d}$ & $28(54)$ & $15(29)$ & $8(15)$ & $1(2)$ \\
\hline & & & $7.5 \%$ & $6.0 \%^{e}$ & $1.9 \%$ & $0 \%$ \\
\hline \multirow[t]{2}{*}{ Control } & 75 & $63 \pm 26$ & $7(9)$ & $33(44)$ & $35(47)$ & $0(0)$ \\
\hline & & & $8.1 \%$ & $3.9 \%$ & $2.9 \%$ & - \\
\hline \multirow[t]{2}{*}{ Exposure $^{*}$} & 34 & $52 \pm 20^{c}$ & $6(18)$ & $17(50)$ & $11(32)$ & $0(0)$ \\
\hline & & & $8.6 \%$ & $4.2 \%$ & $1.5 \%$ & - \\
\hline
\end{tabular}

${ }^{a} P=0.0001$ compared with distribution in controls; $\chi^{2}=34.95$, categories $3(64<x \leqslant 128)$ and $4(x>128)$ were taken together, d.f. $=2$.

$b P<0.0001$ compared with controls; $t=6.39$.

${ }^{c} P=0.03$ compared with controls; $t=2.24$.

$d P=0.004$ compared with exposure group; $t=2.98$.

$e p=0.008$ compared with controls; $t=-2.82$.

-: no blastocysts in these categories.

MI: Mitotic index: the number of mitotic nuclei divided by the total number of nuclei per blastocyst.

*Both sets of experiments were not performed contemporarily, see Table 1.

showed cavitation at day 5 of development in vitro but gave the impression of being smaller than control blastocysts. These day 5 blastocysts were fixed for counting their number of nuclei. Blastocysts were divided into categories (as defined in Materials and Methods and in Table 2). In the control group for ultrarapidly frozen oocytes, $40 \%$ of day 5 blastocysts had passed the fifth cleavage division and had between 32 and 64 nuclei, whereas $47 \%$ of blastocysts had already passed the sixth division and had more than 64 nuclei. Thirteen per cent of blastocysts had less than 32 nuclei. In the group of blastocysts derived from ultrarapidly frozen oocytes, $29 \%$ had between 32 and 64 nuclei but only $17 \%$ had more than 64 nuclei. Most of the blastocysts $(54 \%)$ had fewer than 32 nuclei. These distributions of blastocysts according to their number of nuclei were significantly different $\left(P=0.0001 ; \chi^{2}=34.95\right.$, d.f. $\left.=2\right)$. In addition, the overall mean number of nuclei, irrespective of the different categories, was significantly different: a mean of $70 \pm 34$ nuclei was found in the group of control oocytes versus only $38 \pm 23$ nuclei in the group of blastocysts derived from ultrarapidly frozen oocytes $(P<0.0001 ; t=6.39)$. When comparing the group of blastocysts derived from exposed oocytes with their control group, no differences were found in the distribution of blastocysts over the different categories. The overall mean number of nuclei per blastocyst in the control group was $63 \pm 26$ versus $52 \pm 20$ in the blastocysts from oocytes that were exposed to the freezing solution. This difference in mean number of cells 
Table 3. Chromosomal constitution in first-cleavage mouse embryos derived from oocytes that were ultrarapidly frozen with or exposed to a $3.5 \mathrm{~mol}$ dimethylsulfoxide $\mathrm{I}^{-1} / 0.5 \mathrm{~mol}$ sucrose $\mathrm{I}^{-1}$ solution

\begin{tabular}{lcccl}
\hline & \multicolumn{5}{c}{ Number of embryos (\%) } \\
\cline { 2 - 5 } Treated & Scored & Diploid $^{\mathrm{a}}$ & Aneuploid & Polyploid \\
\hline Control & 35 & $32(91)$ & $1(3)$ & $2(6)$ \\
Ultrarapid freezing* & 31 & $23(74)$ & $1(3)$ & $7(23)$ \\
Control & 47 & $38(81)$ & $4(8.5)$ & $5(10.5)$ \\
Exposure $^{*}$ & 50 & $39(78)$ & $4(8)$ & $7(14)$ \\
\hline
\end{tabular}

${ }^{2}$ Different categories of chromosome ploidy were defined as follows in this study: (1) diploid embryos with two sets of 20 chromosomes; (2) aneuploid embryos with two sets of chromosomes, one of which has 19 or 21 ; (3) polyploid embryos with three or more sets of 20 chromosomes.

*Both sets of experiments were not performed contemporarily.

Ultrarapid freezing: four replicate experiments; exposure: five replicate experiments.

was, however, significant $(P=0.03 ; t=2.24)$. Comparison of the mean number of nuclei between blastocysts from exposed (52) and frozen oocytes (38) indicated a significant difference $(P=0.004 ; t=2.98)$.

\section{Blastocyst mitotic index}

The mitotic index is defined as the number of mitotic nuclei divided by the total number of nuclei per blastocyst. The mitotic index was calculated per category of blastocyst and was declining as the number of nuclei increased (Table 2). The mitotic indices were similar in blastocysts derived from control and frozen-thawed oocytes, except for the category of blastocysts with between 32 and 64 nuclei, where a significantly higher index was found in the experimental group $(P=0.0008 ; t=$ -2.82 ). No significant differences were found between the mitotic indices in blastocysts derived from oocytes exposed to the freezing solution and their controls.

\section{Parthenogenetic activation of oocytes}

The incidence of activation in oocytes following ultrarapid freezing or exposure to the ultrarapid freezing solution was investigated. For each condition, the survival rate was also recorded to indicate that the conditions were not degenerative and not different from the initial findings on survival rate. In the control group, 152 of 160 oocytes were normal $(95 \%)$ and 20 among the $152(13 \%)$ showed signs of activation. They all had the first polar body extruded and one pronucleus present. After ultrarapid freezing, 186 of 200 oocytes $(93 \%)$ survived of which $17(9 \%)$ were activated. After exposure of oocytes to the freezing solution, 174 of 176 oocytes $(99 \%)$ survived of which $20(11 \%)$ were activated. The incidence of activation was not different among the groups of control, exposed or frozen-thawed oocytes.

\section{Chromosome complement of first-cleavage embryos}

Counterparts of the oocytes that were used for chromosome analysis were kept in culture and evaluated for competence of in vitro development. In the group of ultrarapidly frozen oocytes, 158 oocytes were recovered and $112(71 \%)$ survived and were inseminated. Of these, 60 oocytes were prepared for chromosome analysis and 52 remained in culture (53\% two cells, 58 blastocysts). In their control group, 150 oocytes were collected and $138(92 \%)$ were normal and became inseminated. Of these, 72 were prepared for chromosome analysis and 66 remained in culture ( $73 \%$ two-cell formation, $72 \%$ blastocyst formation). In the group of oocytes exposed to the freezing solution, 211 oocytes were recovered and $196(93 \%)$ were normal and became inseminated. Of these, 111 were prepared for chromosome analysis and 85 remained in culture $(70 \%$ two cells, $71 \%$ blastocysts). In their respective control group, 217 oocytes were collected, $208(96 \%)$ were normal and became inseminated. Of these, 111 oocytes were prepared for chromosome analysis and 97 remained in culture ( $72 \%$ two cells, $72 \%$ blastocysts). The number of scoreable preparations and the proportions of diploid, aneuploid and polyploid spreads in each of these groups is shown (Table 3).

In the group of oocytes that were frozen-thawed ultrarapidly, a difference in the ploidy distribution was noted compared with their controls. The percentage of diploid one-cell embryos was $91 \%$ in the control group versus only $74 \%$ in the embryos from frozen-thawed oocytes. This decrease in diploid one-cell embryos from frozen-thawed oocytes was balanced by an increase in polyploid one-cell embryos, being $23 \%$ versus $6 \%$ in the control group. The polyploid embryos were all triploid, except for one that was tetraploid. The incidence of aneuploid embryos was not altered by ultrarapid freezing. In the group of oocytes exposed to the ultrarapid freezing solution, there was no difference in the percentage of diploid, aneuploid or polyploid embryos compared with their control group.

\section{Transfer of embryos to pseudopregnant foster mice}

Counterparts of the oocytes that were used for transfer were kept in culture throughout to assess their development in vitro. Of the 404 oocytes that were recovered after ultrarapid freezing, $319(79 \%)$ survived and were inseminated. Of these, 106 oocytes 
Table 4. Outcome of uterine transfer of day-4 in vitro blastocysts derived from ultrarapidly frozen mouse oocytes

\begin{tabular}{|c|c|c|c|c|}
\hline \multirow[b]{2}{*}{ Treatment } & \multicolumn{2}{|c|}{ Transfer on day 3 of pseudopregnancy } & \multicolumn{2}{|c|}{ Autopsy on day 17 of gestation } \\
\hline & $\begin{array}{l}\text { Total number of } \\
\text { blastocysts transferred } \\
\text { (number of horns) }\end{array}$ & $\begin{array}{l}\text { Number of } \\
\text { blastocysts transferred } \\
\text { to pregnant uterine horns } \\
\text { (number of horns) }\end{array}$ & $\begin{array}{c}\text { Number of } \\
\text { implantations } \\
\text { (\% of transferred } \\
\text { blastocysts) }\end{array}$ & $\begin{array}{c}\text { Number of living } \\
\text { fetuses } \\
\text { (\% of transferred } \\
\text { blastocysts) }\end{array}$ \\
\hline Control & $99(22)$ & $47(12)$ & $27(57)$ & $15(32)$ \\
\hline Ultrarapid freezing ${ }^{*}$ & $78(22)$ & $53(12)$ & $19(36)^{a}$ & $6(11)^{b}$ \\
\hline
\end{tabular}

${ }^{2} \mathrm{P}=0.05$ compared with controls; $\chi^{2}=3.85$, d.f. $=1$.

${ }^{b} P=0.02$ compared with controls; $\chi^{2}=5.19$, d.f. $=1$.

"Ultrarapid freezing: four replicate experiments.

remained in culture and $65 \%$ became two cells of which $55 \%$ progressed to day 5 blastocysts. Of the 213 oocytes allocated to the transfer group, $134(63 \%)$ became two cells and 78 day- 4 blastocysts were transferred to the left uterine horn of 22 pseudopregnant recipients. In the control group, 256 oocytes were collected of which 243 (95\%) were normal and were inseminated. Of these, 73 remained in culture and $75 \%$ became two cells of which $72 \%$ progressed to day- 5 blastocysts. Of the 170 oocytes allocated to the control transfer group, $132(77 \%)$ became two cells and 99 day- 4 blastocysts were transferred to the right uterine horn of the mentioned 22 pseudopregnant females. Twelve of the 22 females became pregnant $(55 \%)$ (Table 4). Autopsy on day 17 of gestation revealed that $57 \%$ of control blastocysts implanted versus only $36 \%$ of blastocysts derived from ultrarapidly frozen oocytes. This difference was significant $\left(P=0.05 ; \chi^{2}=3.86\right)$. In the control group, $32 \%$ of the transferred blastocysts developed to living fetuses. In contrast, only $11 \%$ of blastocysts derived from ultrarapidly frozen oocytes gave rise to living fetuses $\left(P=0.02 ; \chi^{2}=5.19\right)$. Most important, two of the six fetuses obtained showed anatomic abnormalities. One fetus had craniofacial malformations and showed exencephaly while the other fetus was presenting with unclosed abdomen and bowel evisceration.

\section{Discussion}

The data of our present study add useful information to existing data on the developmental competence of mouse oocytes in vitro after ultrarapid freezing with a mixture of $3.5 \mathrm{~mol}$ DMSO $1^{-1}$ and $0.25 \mathrm{~mol}$ sucrose $1^{-1}$ (Surrey and Quinn, 1990). We repeated the experiments of these authors who first described this protocol and extended the study with data on parthenogenetic activation, on chromosome analysis of first-cleavage embryos, on blastocyst cellular content and on developmental competence of blastocysts in vivo. A lowered capacity of development in vitro and in vivo together with anomalies in first-cleavage embryos, in day-5 blastocysts and in living fetuses were reported.

The survival of oocytes after slow freezing is generally high (Chen, 1986; Glenister et al., 1987; Carroll et al., 1989, 1990; Schroeder et al., 1990), but, the percentages of normal oocytes after ultrarapid freezing-thawing are sometimes higher than those achieved by conventional slow freezing techniques (Trounson, 1986; Trounson and Kirby, 1989; Surrey and Quinn, 1990). In addition, in the study reported here, we obtained a high mean percentage of morphologically normal oocytes $(78 \%$ ) even exceeding the $61 \%$ reported by Surrey and Quinn (1990). In contrast, Lai et al. (1990) reported a reduced rate of oocyte normality $(21 \%)$ after ultrarapid freezing with $3.5 \mathrm{~mol}$ DMSO $1^{-1} / 0.25$ mol sucrose $1^{-1}$. Erasmus et al. (1990) obtained $29 \%$ of normal oocytes after ultrarapid freezing with a mixture of $2 \mathrm{~mol} \mathrm{DMSO}{ }^{-1} / 0.25 \mathrm{~mol}$ sucrose $\mathrm{l}^{-1}$ but obtained better results with conventional slow freezing (35\%). A survival reducing factor in these studies may be either the absence of a partial prefreeze dehydration (Lai et al., 1990) or the use of too low concentrations of cryoprotectants (Erasmus et al, 1990). Mazur (1990) stressed that excessive intracellular water may lead to ice crystal formation.

Although a high survival rate was obtained, we reported a mean percentage of two-cells $(59 \%)$ that was lower than in the study of Surrey and Quinn (1990) (77\%). It has been reported that cooling of oocytes (Johnson et al., 1988), exposure to DMSO (Johnson, 1989; Vincent et al., 1990a, b, 1991) or slow freezing with DMSO (Carroll et al., 1990; Wood et al., 1992) can have a zona-hardening and fertilization-decreasing effect. Since exposure to the ultrarapid freezing solution did not decrease fertilization rates in our study, it is possible that the fertilizationdecreasing effect is related to variations in the ultrarapid freezing itself.

In the progress of two cells to blastocysts, a retardation in the formation of day 5 blastocysts from ultrarapidly frozen oocytes and a drastic decrease in the number of nuclei per blastocyst were observed. In the control group, about half of the embryos had passed the sixth cleavage division (more than 64 nuclei), while among blastocysts derived from ultrarapidly frozen oocytes, more than half had not completed the fifth cleavage division (fewer than 32 nuclei). This low number of nuclei is nevertheless compatible with the appearance of the blastocyst. It has been documented that cavitation is not necessarily dependent on the number of cells (Smith and McLaren, 1977; Chisholm et al., 1985; Aziz and Alexandre, 1991) or their ploidy (Snow, 1973). The retardation in growth cannot be explained by a discrepancy in the starting time of the embryo culture of frozen-thawed and freshly collected oocytes since they were inseminated approximately at the same time after hCG and 
were treated in an identical way. Furthermore, blastocysts derived from ultrarapidly frozen-thawed oocytes had not lost their ability to enter mitotic division. Neither is it evident that inappropriate culture conditions may have caused retardation in growth. Kiessling et al. (1991) demonstrated that blastocysts developed in vitro consistently show fewer blastomeres than blastocysts developed in vivo but that this retardation is not reversible by the addition of nutrients or growth factors to the medium. We can, however, not exclude the possibility that there is an interaction between freezing and culture in vitro (Massip et al., 1984) so that frozen-thawed oocytes are more sensitive to culture conditions in vitro. The kinetic retardation observed in blastocysts derived from frozen-thawed oocytes was indeed not observed in blastocysts from oocytes that had been exposed only to the freezing solution.

As developmental failure is often of genetic origin (Edwards, 1986), we examined the chromosome content of embryos before their transit to the two-cell stage. We chose this stage because a bias of the chromosomal spectrum by selection through cleavage cannot yet have been introduced. We found that ultrarapid freezing of oocytes can induce increased polyploidy. Most of the polyploid embryos were triploid. An increase in the level of polyploidy had also been the main drawback for slow freezing of mature oocytes (Wood, 1986; Glenister et al., 1987). The increased incidence of triploid embryos after slow freezing of mouse oocytes was reported to have a digynic origin by nonextrusion of the second polar body (Caroll et al., 1989). In the present study, we could not make a distinction between triploidy of diandric or digynic origin. Another important finding of our study was that, after ultrarapid freezing as after slow freezing, no increase in aneuploidy was observed despite the reported spindle abnormalities with both of these techniques (Wood, 1986; Glenister et al., 1987; Johnson and Pickering, 1987; Pickering and Johnson, 1987; Van der Elst et al., 1988; Aigner et al., 1992). It seems that spindle morphology has little or no predictive value for conditions of either aneuploidy or polyploidy. In a recent study by our team (Aigner et al., 1992), we demonstrated that spindle morphology was widely preserved $\mathrm{l} h$ after recuperation from slow or ultrarapid freezing except for some spindle shape abnormalities. Normal spindle morphology may thus be necessary but not sufficient for genetic integrity of the embryo to result from the oocyte. Owing to the high recovery potential of the spindle of the oocyte, chromosomal analysis of embryos remains a primary requirement. Even more detailed structural chromosome analysis may become necessary since Shaw et al. (1991a) demonstrated that for ultrarapidly frozen two-cell mouse embryos there can be an association between variations in manipulations of the ultrarapid freezing technique and structural chromosome damage.

It is known that parthenogenetic activation of oocytes can give rise to embryos that can pace up very well with preimplantation development up to the compaction stage and then start to show growth retardation (Kaufman, 1991). Since it has been shown that the mature oocyte is sensitive to activation by cryoprotectants (Shaw and Trounson, 1989; Van der Elst et al., 1992), we did not want to exclude the possibility of increased parthenogenetic activation after ultrarapid freezing. Our results, however, do not indicate increased activation after ultrarapid freezing of mouse oocytes. With regard to the use of DMSO, even in high concentrations such as used in ultrarapid freezing, this is in agreement with the study by Shaw and Trounson (1989).

In a final part of our study, we investigated whether it was possible to create normal living fetuses starting from oocytes that were frozen to $-196^{\circ} \mathrm{C}$. The outcome of uterine transfer revealed a lowered rate of implantation and development to living fetuses for blastocysts derived from ultrarapidly frozen oocytes. Moreover, we observed macromorphological abnormalities in some of these fetuses. Since there is a decreased development of frozen-thawed oocytes to the blastocyst stage in our study, transfer at the two-cell stage might have been preferable. However, in a study by Wood et al. (1991) on vitrification of mouse oocytes, a high level of loss after implantation was found despite transfer at the two-cell stage.

According to studies on the relation between genotype and phenotype during development (Kaufman, 1991), our results on chromosomal analysis, blastocyst cell number and fetal abnormalities might be related. It has been stated by Edwards (1986) that the major cause of development failure during the preimplantation stage is of genetic origin. It has also been documented that the number of cells and the early development of mouse embryos after implantation are inversely related to their ploidy (Kaufman, 1991). Triploid mouse embryos were reported to have a delay in cleavage and to be smaller than developmentally matched diploid embryos. More interestingly, triploids frequently display craniofacial abnormalities.

In conclusion, the first promising results on ultrarapid freezing of mature oocytes in terms of the percentages of normal oocytes obtained after thawing and development to the blastocyst stage have to be put into perspective. Chromosomal analysis of firstcleavage embryos revealed an increased percentage of polyploid first-cleavage embryos derived from ultrarapidly frozen oocytes. Counting of nuclei in blastocysts has pointed to a major developmental retardation before the 64-cell stage. After transfer of blastocysts, fetuses with macromorphological abnormalities were present. Whether these observed anomalies are due to technical inadequacies or increased sensitivity of frozen oocytes to culture conditions or whether they are inherent to the procedure of ultrarapid freezing has to be investigated in more detail. The results of the present study do, however, cast some serious doubts on the suitability of the applied ultrarapid freezing technique for mouse oocytes.

This research was funded by a grant (3.0028.91) from the Belgian Fund for Scientific Medical Research. Special thanks are due to F. Winter of the Language Education Centre for his critical correction of the English text.

\section{References}

Aigner S, Van der Elst J, Siebzehnrübl E, Eichenlaub-Ritter U, Todorow S, Wildt L and Van Steirteghem AC (1992) The influence of slow and ultrarapid freezing on the organization of the meiotic spindle of the mouse oocyte Human Reproduction 7 857-864

Al-Hasani S, Diedrich K, van der Ven H, Reinecke A, Hartje M and Krebs D (1987) Cryopreservation of human oocytes Human Reproduction 2 695-700

Aziz $\mathbf{M}$ and Alexandre $\mathbf{H}$ (1991) The origin of the nascent blastocoele in preimplantation mouse embryos: ultrastructural cytochemistry and effect of chloroquine Roux's Archives of Developmental Biology 200 77-85

Caroll J, Warnes GM and Matthews CD (1989) Increase in digyny explains polyploidy after in-vitro fertilization of frozen-thawed mouse oocytes Journal of Reproduction and Fertility $\mathbf{8 5} 489-494$ 
Carroll J, Depypere H and Matthews CD (1990) Freeze-thaw-induced changes of the zona pellucida explains decreased rates of fertilization in frozenthawed mouse oocytes Joumal of Reproduction and Fertility 90 547-553

Chen C (1986) Pregnancy after human oocyte cryopreservation Lancet $i$ 884-886

Chen $C$ (1989) Oocyte freezing. In Clinical in vitro Fertilization pp 113-126 Eds $C$ Wood and A Trounson. Springer-Verlag, London

Chisholm JC, Johnson MH, Warren PD, Fleming TP and Pickering SJ (1985) Developmental variability within and between mouse expanding blastocysts Joumal of Embryology and Experimental Morphology 86 311-336

Edwards RG (1986) Causes of early embryonic loss in human pregnancy Human Reproduction 1 185-198

Erasmus E, Kruger TF, Stander FSH, Menkveld R and Lombard CJ (1990) Comparative analyses of two cryopreservation techniques to freeze mouse oocytes Assisted Reproductive Technology/Andrology 1 331-337

Feichtinger W, Hochfellner $\mathrm{Ch}$ and Ferstl $\mathrm{U}$ (1991) Clinical experience with ultrarapid freezing of embryos Human Reproduction 6 735-736

Glenister PH, Wood MJ, Kirby C and Whittingham DG (1987) Incidence of chromosome anomalies in first-cleavage mouse embryos obtained from frozen-thawed oocytes fertilized in vitro Gamete Research 16 205-216

Gordts S, Roziers P, Campo R and Noto Y (1990) Survival and pregnancy outcome after ultrarapid freezing of human embryos Fertility and Sterility $\mathbf{5 3}$ 469-472

Johnson MH (1989) The effect on fertilization of exposure of mouse oocytes to dimethylsulfoxide: an optimal protocol Journal of in vitro Ferrilization and Embryo Transfer 6 168-175

Johnson MH and Pickering SJ (1987) The effect of dimethylsulphoxide on the microtubular system of the mouse oocyte Development $100313-324$

Johnson MH, Pickering SJ and George MA (1988) The influence of cooling on the properties of the zona pellucida of the mouse oocyte Human Reproduction $3383-387$

Kaufman MH (1983) Early Mammalian Development: Parthenogenetic Studies Ed. MH Kaufman. Cambridge University Press, Cambridge

Kaufman MH (1991) New insights into triploidy and tetraploidy, from an analysis of model systems for these conditions Human Reproduction 6 8-16

Kiessling AA, Davis HW, Williams CS, Sauter RW and Harrison LW (1991) Development and DNA polymerase activities in cultured preimplantation mouse embryos: comparison with embryos developed in vivo Journal of Experimental Zoology 258 34-47

Kolodziej FB, Katzorke Th and Propping D (1991) Cryopreservation of human oocytes and pronuclei embryos - first results Reproduction in Domestic Animals, Zuchthygiene, 24 Jahrestagung über Physiologie und Pathologie der Fortpflanzung, 16 Veterinar-Humanmedizinische Gemeinschaftstagung, Leipzig

Lai AC-H, Ryan JP and Saunders DM (1990) Ultrarapid cryopreservation of mouse oocytes and embryos: effect of cell stage and culture in vitro Assisted Reproductive Technology/Andrology 1 320-330

Magistrini M and Szöllösi D (1980) Effects of cold and of isopropyl-Nphenylcarbamate on the second meiotic spindle of mouse oocytes European Joumal of Cell Biology 22 699-707

Massip A, Van der Zwalmen P, Puissant F, Camus M and Leroy F (1984) Effects of in vitro fertilization, culture, freezing and transfer on the ability of mouse embryos to implant and survive Joumal of Reproduction and Fertility $\mathbf{7 1}$ 199-204

Mazur P (1990) Equilibrium, quasi-equilibrium and nonequilibrium freezing of mammalian embryos Cell Biophysics 17 73-92

Pickering SJ and Johnson MH (1987) The influence of cooling on the organisation of the meiotic spindle of the mouse oocyte Human Reproduction 2 207-216

Pickering SJ, Cant A, Braude P, Currie J and Johnson MH (1990) Transient cooling to room temperature can cause irreversible disruption of the meiotic spindle in the human oocyte Fertility and Sterility 54 102-108

Quinn P, Barros C and Whittingham DG (1982) Preservation of hamster oocytes to assay the fertilizing capacity of human spermatozoa Joumal of Reproduction and Fertility 66 161-168
Schroeder AC, Champlin AK, Mobraaten LE and Eppig JJ (1990) Developmental capacity of mouse oocytes cryopreserved before and after maturation in vitro Journal of Reproduction and Fertility 89 43-50

Shaw JM and Trounson A (1989) Parthenogenetic activation of unfertilized mouse oocytes by exposure to 1,2-propanediol is influenced by temperature, oocyte age and cumulus removal Gamete Research 24 269-279

Shaw JM, Kola I, MacFarlane DR and Trounson AO (1991a) An association between chromosomal abnormalities in rapidly frozen 2-cell mouse embryos and the ice-forming properties of the cryoprotective solution Joumal of Reproduction and Fertility 91 9-18

Shaw IM, Diotallevi $L$ and Trounson AO (1991b) A simple rapid 4.5M dimethylsulfoxide freezing technique for the cryopreservation of one-cell to blastocyst stage preimplantation mouse embryos Reproduction, Fertility and Development $3621-626$

Smith R and McLaren A (1977) Factors affecting the time of formation of the mouse blastocoele Journal of Embryology and Experimental Morphology 41 79-92

Snow MHL (1973) Tetraploid mouse embryos produced by cytochalasin B during cleavage Nature 224 513-515

Surrey ES and Quinn PJ (1990) Successful ultrarapid freezing of unfertilized oocytes Journal of in vitro Fettilization and Embryo Transfer 7 262-266

Tarkowski AK (1966) An air-drying method for chromosome preparation from mouse eggs Cytogenetics 5 394-400

Trounson A (1986) Preservation of human eggs and embryos Fertility and Sterility 1 1-12

Trounson A and Kirby C (1989) Problems in the cryopreservation of unfertilized eggs by slow cooling in dimethyl sulfoxide Fertility and Sterility 52 778-786

Trounson A, Peura A and Kirby C (1987) Ultrarapid freezing: a new low-cost and effective method of cryopreservation Fertility and Sterility $\mathbf{4 8} 843-850$

Trounson A, Peura A, Freemann L and Kirby C (1988) Ultrarapid freezing of early cleavage stage human embryos and eight-cell mouse embryos Fertility and Sterility $49822-826$

Van der Elst J, Van den Abbeel E, Jacobs R, Wisse E and Van Steirteghem A (1988) Effect of 1,2-propanediol and dimethylsulphoxide on the meiotic spindle of the mouse oocyte Human Reproduction 3 960-967

Van der Elst J, Van den Abbeel E, Nerinckx S and Van Steirteghem AC (1992) Parthenogenetic activation pattern and microtubular organisation of the mouse oocyte after exposure to 1,2-propanediol Cryobiology 29 549-562

van Uem JF, Siebzehnrubl E, Scuh B, Koch R, Trotnow S and Lang N (1987) Birth after cryopreservation of unfertilized oocytes Lancet $\mathrm{i}, 752-753$

Vincent C, Pickering SJ and Johnson MH (1990a) The hardening effects of dimethylsulphoxide on the mouse zona pellucida requires the presence of an oocyte and is associated with a reduction in the number of cortical granules present Journal of Reproduction and Fertility 89 253-259

Vincent C, Pickering SJ, Johnson MH and Quick SJ (1990b) Dimethylsulphoxide affects the organisation of microfilaments in the mouse oocyte Molecular Reproduction and Development 26 227-235

Vincent C, Turner K, Pickering SJ and Johnson MH (1991) Zona pellucida modifications in the mouse in the absence of oocyte activation Molecular Reproduction and Development 28 394-404

Whittingham DG (1977) Fertilization in vitro and development to term of unfertilized mouse oocytes previously stored at $-196^{\circ} \mathrm{C}$ Journal of Reproduction and Fertility $4989-94$

Wood MJ (1986) An increased incidence of polyploidy in embryos derived from frozen oocytes. In Workshop on Embryos and Oocytes Freezing (Reports), pp 4I-5I Eds Y Menezo and Ch Mérieux. Collection Fondation Marcel Mérieux, Annecy

Wood MJ, Carroll JC, Whittingham DG, Barros C and Candy C (1991) Vitrification of mouse and hamster oocytes. Cryobiology 28 Abstract 12

Wood MJ, Whittingham DG and Lee SH (1992) Fertilization failure of frozen mouse oocytes is not due to premature cortical granule release Biology of Reproduction 46 1187-1195 